\title{
Energy Minimization and Observability Maximization in Multi-Hop Wireless Sensor Networks
}

\author{
Didier Georges* \\ * Grenoble INP / Univ Grenoble 1 / Univ Grenoble 3 / CNRS, \\ GIPSA-lab UMR 5216, F-38402 Saint Martin d'Hères, (e-mail: \\ didier.georges@gipsa-lab.grenoble-inp.fr).
}

\begin{abstract}
This paper is devoted to large-scale monotoring by using multi-hop wireless sensor networks. Environmental applications such as pollution monitoring are considered in this paper. We consider the problem of minimizing the overall energy comsuption of the network while maximizing observability, with a receding horizon control strategy. The measure of observability is defined by the transient observability gramian associated to a finite-dimensional model of the PDE governing the pollution dynamics. Some numerical results based on a realistic case study demonstrate the effectiveness of the proposed approach.
\end{abstract}

Keywords: Sensor networks, optimal routing, pollution monitoring, transient observability gramian, optimal location of sensors, receding horizon control.

\section{INTRODUCTION}

Sensor networks are recognized for being well adapted for measurement, monitoring, tracking of distributed physical phenomena, such as environmental phenomena (weather, seismic events, wildfires, air, soil or river pollution, sound or vibration monitoring ...) or complex industrial processes (in the chemical industry for example). In this paper, we consider sensor networks defined as a collection of wireless embedded sensors disseminated on the field of interest, and more and more with mobility capabilities (Cortes et al. (2004)). Large-scale deployment of such ad hoc networks relies on the availability of cheap, small-size embedded sensors with sufficient energy autonomy. More specifically, we consider the main environmental issue of monitoring air pollution. Some approaches have been proposed for that purpose on the basis of a regular grid of sensors (Ghanem et al. (2004)).

In this paper, we consider the case of randomly distributed wireless sensors. Random distribution of the embedded sensors generates several main issues (Woo et al. (2003)), such as poor or unstable wireless communication quality inducing bandwith limitations and energy autonomy (Woo et al. (1998)). Many researches have been devoted in the last decade to the design of routing protocols to face sensor network issues (see Akkaya (2005)), Heinzelman et al. (2000)). Some optimal routing strategies based on a dynamical model of energy consumptiom of each node of the network have been also recently proposed in order to increase the network life (Wu et al. (2005)).

On the other hand, there are still relatively few research results on application-dependant approaches. See for example (Cortes et al. (2004)) for the coverage control problem for mobile sensing networks, but also some approaches dedicated to chemical plume tracing with robot swarms (Zarzhitsky et al. (2005)).

In this paper, a new application-dependant dynamic routing procedure under wireless communication constraints is proposed. It can be applied to a large class of estimation or physical monitoring problems governed by partial differential equations. The here-proposed approach is based on an observability index based on the observability gramian (Georges (1995)) and a model of the energy consumption of each node. The goal is to minimize the energy consumption (to ensure the network life), while maximizing the observability index.

The paper is now organized as follows: Section 2 is devoted to some backgrounds on air pollution modelling and monitoring. Section 3 is dedicated to the formulation of the horizon control strategy. Some numerical results are provided in section 4 . Finally some conclusions and perspectives are also provided.

\section{AIR POLLUTION MODELING}

\subsection{PDE Governing Pollution Dynamics}

Air pollution (without chemical reaction) may be well modeled (see Tirabassi (1989) and Zannetti (1990)) on a $2 \mathrm{D}$ domain $\Omega$ by an advection-diffusion partial differential equation of the form:

$$
\begin{aligned}
\frac{\partial z}{\partial t}+U_{x}(x, y, t) \frac{\partial z}{\partial x}+U_{y}(x, y, t) \frac{\partial z}{\partial y} & =\frac{\partial}{\partial x} K \frac{\partial z}{\partial x}+\frac{\partial}{\partial y} K \frac{\partial z}{\partial y} \\
& +\delta\left(x-x_{s}, y-y_{s}\right) S(t)
\end{aligned}
$$

where $z(x, y, t)$ is the concentration of a chemical species in the air (the pollutant), $\left(U_{x}, U_{y}\right)^{T}$ is the vector of the 
wind velocities which is supposed to be known (based on weather forecast or measurements), $K$ is a constant diffusion coefficient which is supposed to be known, and $S(t)$ is the source of pollution located at position $\left(x_{s}, y_{s}\right)$.

Some initial conditions $z(x, u, t=0)=z_{0}(x, y)$ and some boundary conditions (such as Pearson's conditions) on the domain boundary $\partial \Omega$ have to be provided:

$$
\begin{array}{r}
\frac{\partial z}{\partial t}(x, y, t)+\mu_{x} \frac{\partial z}{\partial x}(x, y, t) \\
+\mu_{y} \frac{\partial z}{\partial y}(x, y, t)=0, \forall(x, y) \in \partial \Omega,
\end{array}
$$

where $\left(\mu_{x}, \mu_{y}\right)$ is the equivalent celerity vector.

\subsection{Finite-Dimensional Modelling}

A finite-dimensional state-space representation may be derived by using a discretization scheme, such as the finitedifference method defined on $2 \mathrm{D}$ regular grid:

$$
\begin{gathered}
\frac{\partial z}{\partial x}=\left\{\begin{array}{l}
\frac{z(x, y, t)-z\left(x-h_{x}, y, t\right)}{h_{x}}, \text { if } U_{x}>0 \\
\frac{z\left(x-h_{x}, y, t\right)-z(x, y, t)}{h_{x}}, \text { if } U_{x}<0
\end{array}\right. \\
\frac{\partial^{2} z}{\partial x^{2}}=\frac{z\left(x+h_{x}, y, t\right)-2 z(x, y, t)+z\left(x-h_{x}, y, t\right)}{h_{x}^{2}}
\end{gathered}
$$

where $h_{x}$ is the discretization step. The same scheme applies for the discretization in the $y$ coordinate with a discretization step $h_{y}$.

The time discretization scheme is defined by:

$$
\frac{\partial z}{\partial t}=\frac{z(x, y, t+T)-z(x, y, t)}{T}
$$

where $T$ is the sampling time, which has to chosen small enough to ensure numerical stability.

Finally a discrete-time state representation can be easily derived by using the previous discretization scheme:

$$
\begin{array}{r}
Z_{n+1}=A_{n} Z_{n}+B(\theta) S_{n} \\
Y_{n}=C\left(V_{L}, \alpha\right) Z_{n}
\end{array}
$$

where $Z_{n}$ is the $N_{x} \times N_{y}$-vector of the values of the concentration $z, z\left(x_{i}, y_{j}, n\right)$ defined at time $n T$, at each location $\left(x_{i}=i h_{x}, y_{j}=j h_{y}\right)$ of the $N_{x} \times N_{y}$-node grid, $S_{n}$ is the source magnitude at time $n T$ and, $Y_{n}$ is the $M$ vector of measurements produced by the $M$ sensors of the network.

$A_{n}$ denotes the state matrix which depends on time because the wind velocities may vary due to changing weather conditions. $C\left(V_{L}, \alpha\right)$ is the observation matrix depending on both the location vector $V_{L}$ of the $M$ sensors and $\alpha$, the vector of activity state of the $M$ sensors. $0 \leq \alpha^{i} \leq 1$ represents the activity percentage of the sensor $i$ over a given time period: $\alpha^{i}=1$, when the sensor $i$ is always active. This particular metric corresponds to the amount of measurements and computation performed to get a reliable value of the pollutant at the node location over the given time period. $\alpha^{i}$ can also be viewed as a level of confidence in the measured value.
By using for instance a bilinear approximation technique, each sensor output may be expressed as follows:

$$
Y_{n}^{i}=\alpha^{i} F_{i}\left(V_{L}^{i}\right) Z_{n}
$$

where $F_{i}$ is a bilinear function of the location $V_{L}^{i}$ of the sensor $i$. $\theta$ denotes the unknown location $\left(x_{s}, y_{s}\right)^{T}$ of the source $S_{n}$ to be determined together with the source magnitude.

\subsection{Pollution Monitoring via State and Source Observer}

Under the assumption that the source is slowly timevarying and with the goal of determining both the magnitude and the location of the source together with the distribution of the pollutant, an augmented model may be defined:

$$
\begin{array}{r}
Z_{n+1}=A_{n} Z_{n}+B\left(\theta_{n}\right) S_{n} \\
S_{n+1}=S_{n} \\
\theta_{n+1}=\theta_{n} \\
Y_{n}=C\left(V_{L}, \alpha\right) Z_{n}
\end{array}
$$

An extended optimal LQ estimator may be then derived to estimate both the states, the source magnitude and the source location:

$$
\begin{array}{r}
\hat{Z}_{n+1}=A_{n} \hat{Z}_{n}+B\left(\theta_{n}\right) \hat{S}_{n}+L_{n}^{1}\left(Y_{n}-C\left(V_{L}, \alpha\right) \hat{Z}_{n}\right) \\
\hat{S}_{n+1}=\hat{S}_{n}+L_{n}^{2}\left(Y_{n}-C\left(V_{L}, \alpha\right) \hat{Z}_{n}\right) \\
\hat{\theta}_{n+1}=\hat{\theta}_{n}+L_{n}^{3}\left(Y_{n}-C\left(V_{L}, \alpha\right) \hat{Z}_{n}\right)
\end{array}
$$

where the $L_{n}^{i}$ 's represent the observer gains obtained from the discrete-time Riccati equation of the extended optimal LQ estimator. We denote $L_{n}=\left(\begin{array}{c}L_{n}^{1} \\ L_{n}^{2} \\ L_{n}^{3}\end{array}\right)$. (For more details on the use of extended Kalman estimator for monitoring, (see Torres et al. (2005) for instance). The observer gain matrix is given by

$$
L_{n}=A_{a}\left(\hat{\theta}_{n}, \hat{S}_{n}\right) M_{n} C_{a}^{T}(\alpha)\left(R+C_{a}(\alpha) M_{n} C_{a}^{T}(\alpha)\right)^{-1}(15)
$$

The associated discrete-time Riccati equation is given by:

$$
\begin{array}{r}
A_{a}\left(\hat{\theta}_{n}, \hat{S}_{n}\right) M_{n} A_{a}^{T}\left(\hat{\theta}_{n}, \hat{S}_{n}\right)-M_{n+1}-A_{a}\left(\hat{\theta}_{n}, \hat{S}_{n}\right) M_{n} C_{a}^{T}(\alpha) \\
\times\left(R+C_{a}(\alpha) M_{n} C_{a}^{T}(\alpha)\right)^{-1} C_{a}(\alpha) M_{n} A_{a}^{T}\left(\hat{\theta}_{n}, \hat{S}_{n}\right)+Q=0, \\
M_{0}=S_{0},
\end{array}
$$

where $Q$ is the covariance matrix of the state noise, $R$ is the covariance matrix of the observation noise, and $S_{0}$ is the covariance matrix of the initial state.

The performance of this observer strongly relies on the observability properties of the augmented system (8)-(11). In particular, observability may be checked by the so-called transient observability gramian $P_{N_{o}}$ of the augmented system (8)-(11) defined as the solution of the following recursive discrete-time Lyapunov equation:

$$
\begin{array}{r}
A_{a}^{T}\left(\hat{\theta}_{n}, \hat{S}_{n}\right) P_{n} A_{a}\left(\hat{\theta}_{n}, \hat{S}_{n}\right)-P_{n+1}+C_{a}^{T}(\alpha) C_{a}(\alpha)=0 \\
P_{0}=0_{N+3 \times N+3},
\end{array}
$$


where $A_{a}\left(\hat{\theta}_{n}, \hat{S}_{n}\right)$ and $C_{a}(\alpha)$ denote the state-space matrix and the output matrix of the linearized augmented system around the trajectory $\hat{Z}_{n}, \hat{S}_{n}, \hat{\theta}_{n}$ of the observer. $N_{o}$ is an observation horizon.

$$
\begin{array}{r}
A_{a}\left(\hat{\theta}_{n}, \hat{S}_{n}\right)=\left(\begin{array}{ccc}
A_{n} & B\left(\hat{\theta}_{n}\right) & \frac{\partial B}{\partial \theta}\left(\hat{\theta}_{n}\right) \hat{S}_{n} \\
0_{1 \times N} & 1 & 0_{1 \times 2} \\
0_{2 \times N} & 0_{2 \times 1} & I_{2}
\end{array}\right) \\
C_{a}(\alpha)=\left(\begin{array}{lll}
C\left(V_{L}, \alpha\right) & 0_{M \times 1} & 0_{M \times 2}
\end{array}\right)
\end{array}
$$

with $N=N_{x} \times N_{y}$.

A so-called persistence of excitation condition is given by the fact that the solution $P_{N_{o}}$ of (17) is positive semidefinite. This condition is equivalent to a detectability condition of the linearized augmented system around the trajectory $\hat{Z}_{n}, \hat{S}_{n}, \hat{\theta}_{n}$ of the observer, defined by

$$
\begin{array}{r}
X_{n+1}=A_{a}\left(\hat{\theta}_{n}, \hat{S}_{n}\right) X_{n} \\
Y_{n}^{l}=C_{a}(\alpha) X_{n}
\end{array}
$$

The observability gramian $P_{N_{o}}$ is related to the energy $E_{o}$ of the output $Y_{n}^{l}$ over the interval $\left[0, N_{o}\right]$ generated by the initial state $X_{0}$ of the linearized augmented system:

$$
E_{o}=\sum_{k=0}^{N_{o}}\left\|Y_{k}^{l}\right\|^{2}=X_{0}^{T} P_{N_{o}} X_{0} .
$$

As a consequence $P_{N_{o}}$ may be used as a measure of the "level of observability" induced by a particular choice or location of sensors in the system. An optimal location of sensors will consist for instance in maximizing the minimum singular value of $P_{N_{o}}$ (see Georges (1995)) or the trace of $P_{N_{o}}$, which represents the sum of the singular values of $P_{N_{o}}$.

\section{FORMULATION OF THE OPTIMAL ROUTING PROBLEM}

\subsection{Structure and Model of the Sensor Network}

We consider sensor networks consisting of $M$ identical sensors. One of them is a base station. Each sensor is equiped with a wireless Ethernet interface, a GPS, a solar cell and a battery ensuring a limited energetic autonomy and some measurement equipment adapted to the chemical species to be measured. Data communication is based on a multi-hop protocol. The Network nodes (sensors) cooperate to forward data packets for each other to allow communication over multiple hops between nodes not directly within wireless transmission range of one another. The $M$ sensors are not supposed to be uniformly distributed on the field, since the sensors are supposed to be disseminated by aircraft. We arbitrarly decide that the node 1 is the base station. The base station is a master station in the sense that it periodically (with a constant sampling rate) sends some measurement requests to the active sensor nodes in order to update the monitoring information. We also suppose that the energy of the base station is not constrained.

We suppose that the protocol includes a mechanism of route discovery which consists in establishing a connectivity table, i.e. for each node, the set of the reachable nodes via the wireless connection, which defines the set of all possible routes from or to the base station to or from the all reachable nodes. The connectivity table is a $M \times M$ network adjacence matrix of elements $\beta_{i j} \in\{0,1\}$, where $\beta_{i j} \in\{0,1\}$ is equal to 1 when the node $j$ is reachable by the node $i$. According to the connectivity matrix, we can derive, for each node $i$, the set of the nodes connected to $i: C_{i}=\left\{j, j=1, \ldots, M, j \neq i / \beta_{i j}=1\right\}$.

We assume that each node can reach the base station (if it is not the case, the node may be removed from the list). The connectivity is supposed to be checked periodically. From this connectivity matrix, the incidence matrix $M_{I}$ of the network may be derived. If $\delta$ is the $M_{c}$ vector of the $\delta^{i j}$ 's, where $\delta^{i j}$ denotes the average number of packets routed from the node $i$ to the node $j$ and $M_{c}=\sum_{i, j} \beta_{i j}=\sum_{i=1}^{M} \operatorname{card}\left(C_{i}\right)$, we get the following model of communications links:

$$
M_{I} \delta+d \alpha-H d_{0}=0_{M_{c} \times 1},
$$

where $d$ is the maximum number of packets transmitted by any sensor node (routing information and measurement packets), $d$ is supposed to be fixed. $H=(1, \ldots, 0)^{T}$, and $d_{0}$ is the maximum number of packets received by the base station. Since all the packets are supposed to converge towards the base station, $d_{0}=\sum_{i=1}^{M} d \alpha^{i} \cdot \alpha^{i}$ is introduced to take into account the fact that the sensor $i$ may be less active due to energy starvation.

We also suppose that the amount of energy needed to send a packet of the measurement data, status and routing data between the node $i$ and the node $j$ is available through a priori experiments at each sampling time on request of the base station and is given by a coefficient $k_{i j}^{s}>0 . k_{i j}^{s} 0$ depends on the distance $d_{i j}$ between the node $i$ and the node $j$, since the emission power needed to reach the node $j$ increased as a function of the distance $d_{i j}$. On the other hand, the amount of energy needed to receive a packet from the node $j$ is given by a coefficient $k_{i j}^{r}>0$. A model of energy consumption at each node $i, i=1, \ldots, M$ is then:

$$
\begin{aligned}
e_{n+1}^{i}= & e_{n}^{i}-k_{i i} \alpha^{i}-\sum_{j \in C_{i}}\left[k_{i j}^{s} \delta^{i j}+k_{i j}^{r} \delta^{j i}\right]+E_{n}^{i}-p_{n}^{i}(23) \\
& e_{0}^{i}=e_{I}^{i} \\
& \underline{e}^{i} \leq e_{n}^{i} \leq \bar{e}^{i} \\
& p_{n}^{i} \geq 0
\end{aligned}
$$

where $e_{n}^{i}$ is the available energy of node $i$ at sampling time $n T, e_{I}^{i}$ is the initial available energy stored in the node battery, and $E_{n}^{i}$ is the energy provided by the solar cell of the sensor $i$ at the sampling time $n T$. The coefficient $k_{i i}$ correspond to the energy consumed by the node $i$ when it collects and computes its own air pollution measurement. $\underline{e}^{i}$ and $\bar{e}^{i}$ are the discharge and full battery bounds, respectively. $p_{n}^{i}$ is a energy "spill" variable to take into account the full battery state. 


\subsection{A Receding Horizon Optimal Control Problem}

In this section, we propose a procedure to update the routing at each time sampling $n T$, in order to minimize the overall energy consumption of the network and maximize observability, over a receding horizon $\left(n+N_{p}\right) T$, i.e. the optimal sensor commitment over the period $\left[n T,\left(n+N_{p}\right) T\right]$. A trade-off must be found between reducing the number of active nodes (in order to reduce the energy consumption) and satisfying a minimal level of observability with a large enough number of active sensors well located on the field. This trade-off is defined by the cost function (27).

The optimal routing problem (ORP) may be formulated as follows:

$$
\begin{array}{r}
\min _{\delta_{l}, \alpha_{l}, d_{l}^{L}, p_{l}} \sum_{l=n}^{n+N_{p}-1}\left\{\sum _ { i = 1 } ^ { M } \left(k_{i i} \alpha_{l}^{i}\right.\right. \\
\left.+\sum_{j \in C_{i}}\left[k_{i j}^{s}\left(d_{i j}\right) \delta_{l}^{i j}+k_{i j}^{r} \delta_{l}^{j i}\right]\right)+K_{p} \sum_{j=1}^{M}\left(p_{l}^{j}+d_{j l}^{L}\right) \\
\left.-\sigma \operatorname{trace}\left(P\left(n-N_{o}, n, \alpha_{l}\right)\right)\right\} \\
s . t \\
e_{l+1}^{i}=e_{l}^{i}-k_{i i} \alpha_{l}^{i}-\sum_{j \in C_{i}}\left[k_{i j}^{s} \delta_{l}^{i j}+k_{i j}^{r} \delta_{l}^{j i}\right]+\hat{E}_{l}^{i}-p_{l}^{i} \\
M_{I} \delta_{l}+d \alpha_{l}-H \sum_{M_{c} \times 1}^{M} d \alpha_{l}^{i}-d_{l}^{L}=0_{0}=e_{I}^{i}, \\
e^{i} \leq e_{l}^{i} \leq \bar{e}^{i}, \\
0 \leq \delta_{l}^{i j} \leq \bar{\delta}^{i j}\left(d_{i j}\right), 0 \leq \alpha_{l}^{i} \leq 1, d_{l}^{L} \geq 0, p_{l}^{i} \geq 0,
\end{array}
$$

where $\sigma>0$ is the "trade-off coefficient" and $K_{p}>0$ is a large enough penalty coefficient, $P\left(n-N_{o}, n, \alpha_{l}\right)$ is the observability gramian, solution of the discrete-time Lyapunov equation (17) computed on the time interval $\left[\left(n-N_{o}\right) T, n T\right], \hat{E}_{l}^{i}$ is a prediction (except at current time $n T$ ) of the amount of energy provided by the solar cell (depending on both night or day time and weather conditions). $e_{n}^{i}$ is the amount of energy stored in the battery, available for the node $i$ at $n T . \bar{\delta}^{i j}\left(d_{i j}\right)$ is the maximum number of packets sent by the node $i$ to the node $j$ at every sampling time (a data flow rate limit), which will depends on the inverse of the distance $d_{i j}$ since the transmission rate is affected by the quality of the wireless link. $d_{l}^{L}$ is the $M$ vector of packet losses at each node. Packet losses may occur, when some links are congestioned due to data flow rate limits.

In a receding horizon strategy, only the first control input of the optimal control sequence defined on the time interval $\left[n T, n T+N_{p}-1\right]$ is applied to the system at each sampling time $n T$ (see (Mayne et al. (2000)) for example for a detailed description of predictive control strategy based on receding horizon).

The transient observability gramian $P\left(n-N_{o}, n, \alpha_{l}\right)$ has to be computed on a past time interval $\left[\left(n-N_{o}\right) T, n T\right]$ due to the causality condition on the output, since the solution of the extended Kalman filter (based on the sensor measurements) is needed for computing (17).
(ORP) is a linear-quadratic optimization problem since it can be easily shown that $\operatorname{trace}\left(P\left(n-N_{o}, n, \alpha_{l}\right)\right)$ is a quadratic functional of the $\alpha_{l}^{i}$, s. A decompositioncoordination algorithm based on an augmented Lagrangian approach very similar to the one proposed in (Georges (1995)) has been used. Due to the lack of place, this algorithm is not detailed in this paper.

\section{NUMERICAL RESULTS}

The monitoring problem consists in estimating both the magnitude and the location a pollution source together with the distribution of the pollution over a domain $[2000 \mathrm{~m}, 2000 \mathrm{~m}]$. The advection-diffusion partial differential equation governing the dynamics of the pollution, together with the parameters of the time-explicit finitedifference scheme, are given in Table 1. Fig. 2 gives a typical distribution of the singular values of the transient observability gramian associated to the augmented system (8)-(11).

Table 1. EDP and discretization scheme parameters.

\begin{tabular}{ccccccc}
$\begin{array}{c}U_{x} \\
(\mathrm{~m} / \mathrm{s})\end{array}$ & $\begin{array}{c}U_{y} \\
(\mathrm{~m} / \mathrm{s})\end{array}$ & $\begin{array}{c}K \\
\left(\mathrm{~m}^{2} / \mathrm{s}\right)\end{array}$ & $\begin{array}{c}x_{s}, y_{s} \\
(\mathrm{~m})\end{array}$ & $N_{x}$ & $N_{y}$ & $\begin{array}{c}T \\
(\mathrm{~s})\end{array}$ \\
\hline 5 & 5 & 1 & 500,500 & 10 & 10 & 600 \\
\hline
\end{tabular}

However in this paper attention is only paid to the energyaware routing problem with maximization of observability. In order to illustrate the proposed approach, we consider the randomly generated sensor network depicted in Fig. 3. The red sensor is the base station, while the dotted lines mean that a connection may be established between the considered nodes. The main characteristics of the network are given in Table 2. The maximum distance allowing a wireless connection is large to increase the routing complexity. The $k_{i i}$ 's, $k_{i j}^{s}$ and $k_{i j}^{r}$ 's are some explicit functions of the distance between each connected node, as far as the maximum reachable data rates $\bar{\delta}^{i j}$, and are not detailed in this paper.

Table 2. Main characteristics of the network.

\begin{tabular}{ccc} 
Number of sensors & number of links $\delta_{i j}$ & connectivity \\
\hline 50 & 296 & $500 \mathrm{~m}$ \\
\hline
\end{tabular}

In order to illustrate the trade-off between energy minimization and observability maximization, we consider the two following strategies corresponding to two different values of the trade-off coefficient $\sigma$ : With $\sigma=2$, we focus on observability maximization, while with $\sigma=0.1$, energy consumption minimization is reinforced. Fig. 4 and 5 provide the simulation results obtained by applying the receding horizon optimal control strategy (ORP), with a receding horizon of 6 hours to the sensor network over a 24 hour period. The optimization parameters are given by the Table 3, with $\sigma=2$. Both the energy dynamics of all the sensor batteries and the network traffic vary according to the solar energy received by each sensor. As expected, the observability index strongly depends on the health of the network. We can notice that the data traffic increases on some critical links of the networks when the level of available energy is high. As also expected, Fig. 6 and 7 demonstrate a completely different behaviour of 
the network with $\sigma=0.1$. The observability index is reduced to a minimum with this strategy, while the energy consumption is dramatically reduced.

Table 3. Optimization parameters.

\begin{tabular}{ccccccc}
$N_{p}$ & $N_{o}$ & $\sigma$ & $K_{p}$ & $\underline{e}^{i}$ & $\bar{e}^{i}$ & $e_{0}^{i}$ \\
\hline 6 & 3 & $0.1,2$ & 0.1 & 1 & 20 & 15 \\
\hline
\end{tabular}

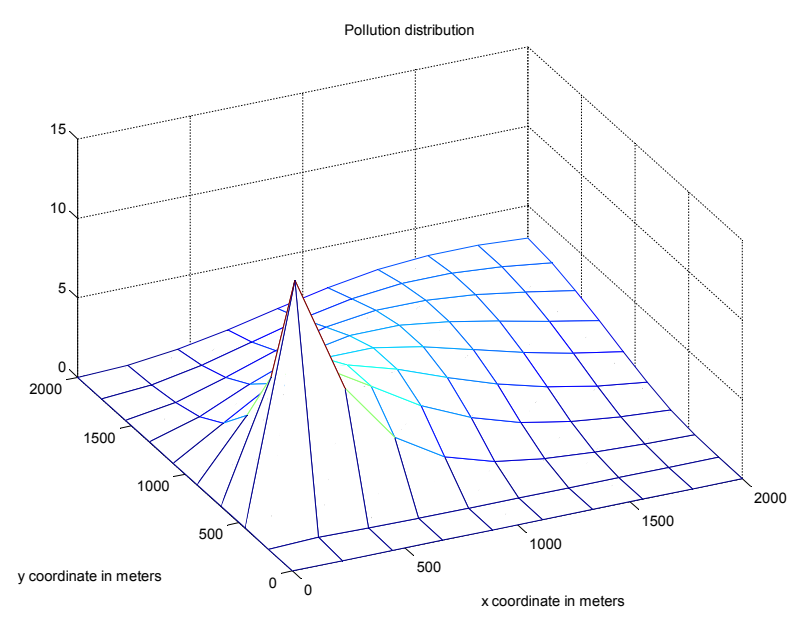

Fig. 1. Distribution of the pollutant at equilibrium.

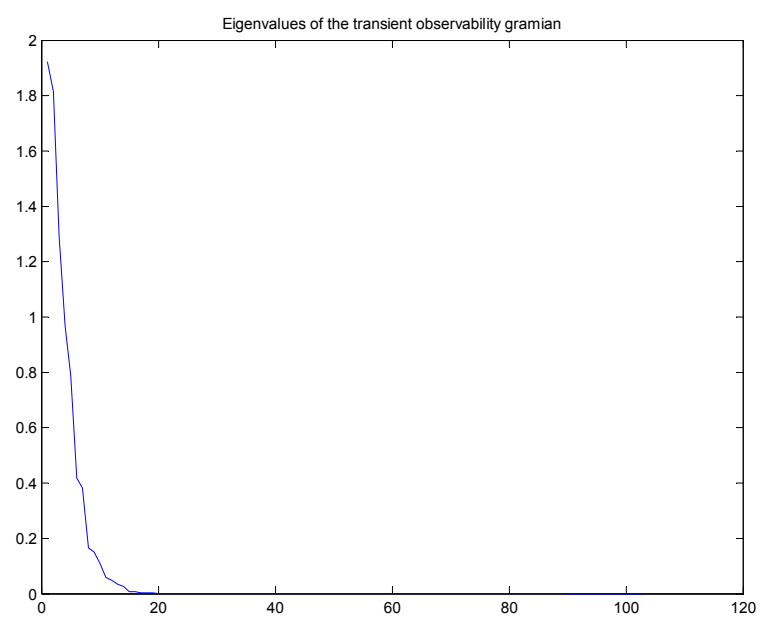

Fig. 2. An example of the observability gramian singular value distribution when all the sensors are supposed to be active. Notice that only $20 \%$ of the singular values are greater than 0.01 , which means that $80 \%$ of the states are "weakly" observable.

\section{CONCLUSIONS AND PERSPECTIVES}

In this paper, a receding horizon strategy was proposed to manage the energy consumption of sensor networks, while maximizing the monitoring capabilities defined by

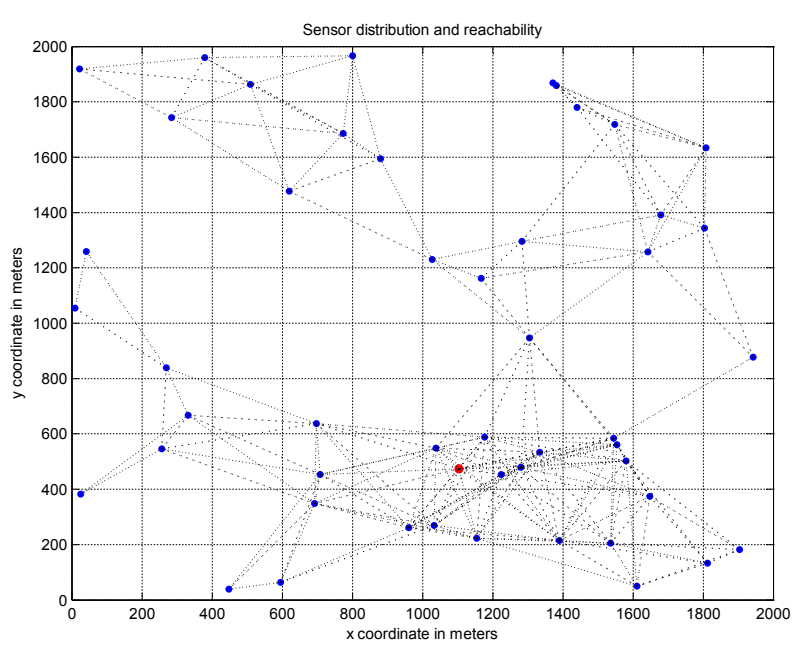

Fig. 3. A randomly distributed sensor network.

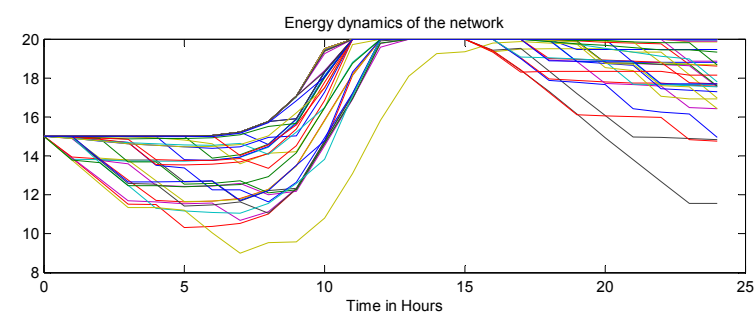

Traffic of the network

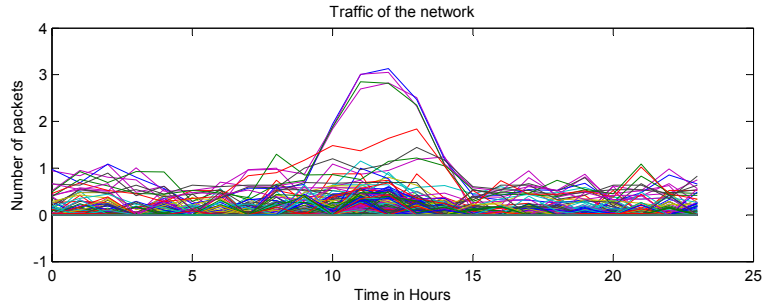

Fig. 4. Energy dynamics and data traffic $-\sigma=2$.

an observability index based on the trace of an appropriate transient observability gramian. Numerical results are provided which demontrate the effectiveness of this control strategy. Future works will be devoted to the extension of this approach to the case of mobile sensor networks with the same goal of managing the trade-off between energy minimization and observability maximization.

\section{REFERENCES}

Akkaya, K., and Younis, M. (2005). A survey on routing protocols for wireless sensor networks. Ad Hoc Networks, Volume 3, Issue 3, Pages 325-349, 2005.

Cortes, J., Martinez, S., Karatas, T., and Bullo, F. (2004). Coverage control for mobile sensing networks. IEEE Transactions on Robotics and Automation, Volume: 20 Issue: 2, April 2004. 

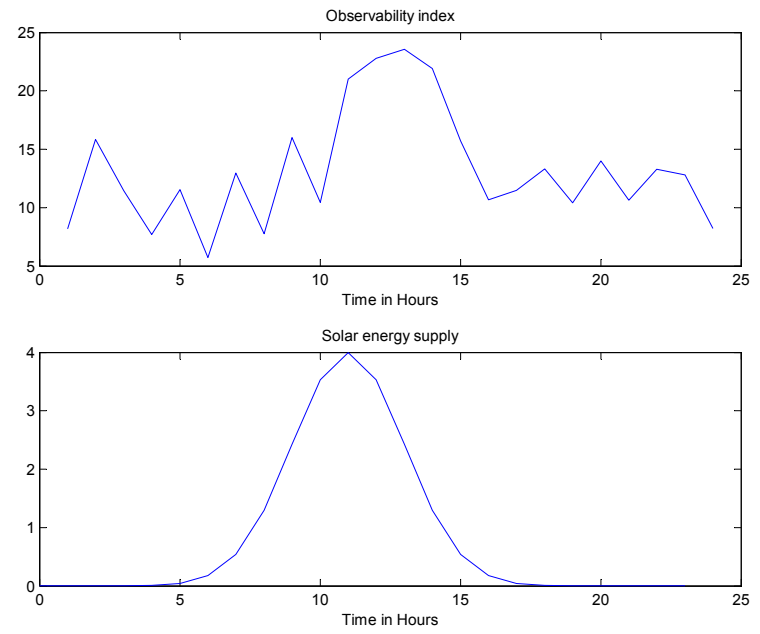

Fig. 5. Observability index and solar energy supply $-\sigma=2$.
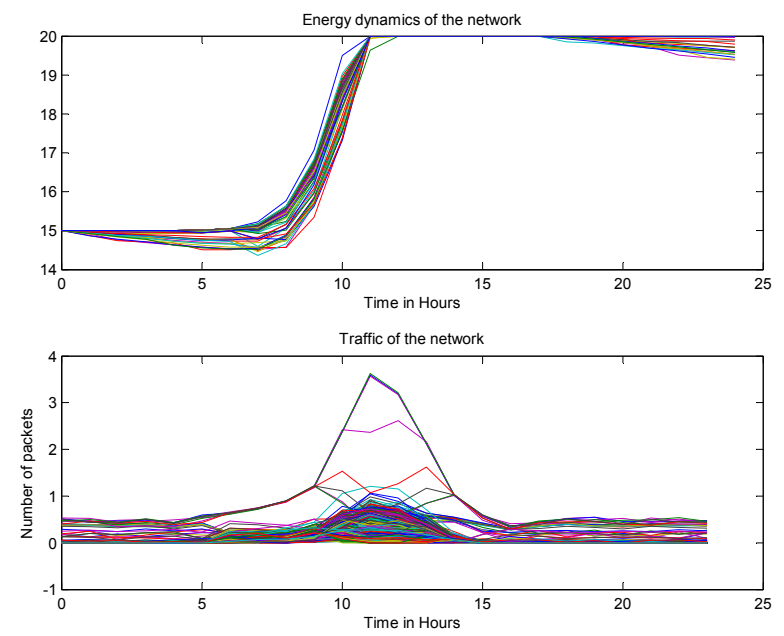

Fig. 6. Energy dynamics and data traffic $-\sigma=0.1$.

Georges, D. (1994). Optimal Unit Commitment in Simulations of Hydrothermal Power Systems: An Augmented Lagrangian Approach. Simulation Practice and Theory, 1(4):155-172, March 1994.

Georges, D. (1995). The use of observability and controllability gramians or functions for optimal sensor and actuator location in finite-dimensional systems. Proceedings of the 34th IEEE Conference on Decision and Control, 1995.

Ghanem, M., Guo Y., Hassard, J., Osmond, M., Richards, M. (2004). Sensor Grids For Air Pollution Monitoring. In Proc. 3rd UK e-Science All Hands Meeting, 2004.

Heinzelman, W. R., Chandrakasan, A., and Balakrishnan, H. (2000). Energy-Efficient Communication Protocol for Wireless Microsensor Networks. Proceedings of the Hawaii International Conference on System Sciences, January 4-7, 2000, Maui, Hawaii.
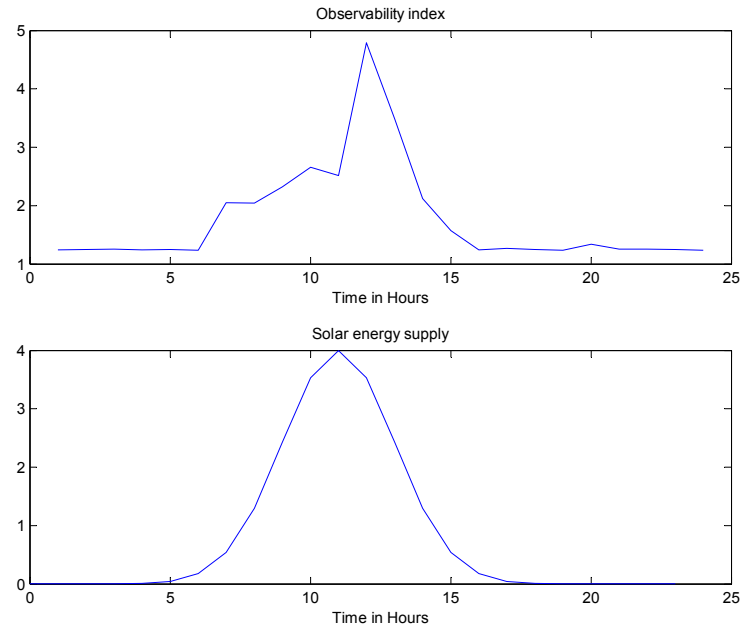

Fig. 7. Observability index and solar energy supply $\sigma=0.1$.

Mayne, D. Q., Rawlings J. B., Rao C. V., and Scokaert, P. O. M. (2000). Constrained Model Predictive Control: Stability and optimality. Automatica, 36(6):789-814, June 2000.

Tirabassi, T. (1989). Analytical air pollution advection and diffusion models Water, Air, \& Soil Pollution, Volume 47, Numbers 1-2, september 1989.

Torres, L., Besancon, G., and Georges, D. (2008). A collocation model for water-hammer dynamics with application to leak detection. Proceedings of the 47th IEEE Conf Decision and Control - 47th IEEE Conference on Decision and Control, CDC 2008, Mexique (2008) [hal00348367].

Woo, A., Tong, T., and Culler, D. (2003). Taming the Underlying Challenges of Reliable Multihop Routing in Sensor Networks. SenSys'03, November 5-7, 2003, Los Angeles, California, USA.

Woo, M., Singh, S., and Raghavendra, C. S. (1998). Poweraware routing in mobile ad hoc networks. Proceedings of the 4th annual ACM/IEEE international conference on Mobile computing and networking, Dallas, Texas, United States, Pages: 181 - 190, 1998.

Wu, X., and Cassandras, C. G. (2005). A Maximum Time Optimal Control Approach to Routing in Sensor Networks. Proceedings of the 44th Conference on Decision and Control and the European Control Conference 2005, Seville, Spain, Dec. 12-15, 2005.

Zannetti, P. (1998). Air Pollution Modelling. Theories, Computational Methods and Available Software. Computational Mechanics Publications, Southampton, Boston, Van Nostrand Reinhold, New York, 1990.

Zarzhitsky, D., Spears, D. F., and Spears W. (2005). Distributed Robotics Approach to Chemical Plume Tracing. Proceedings of the IEEE/RSJ International Conference on Intelligent Robots and Systems (IROS05). 